AN. MEd. INTERNA (Madrid) Vol. 18, N. $^{\circ} 10$, pp. 525-528, 2001

\title{
Trombosis de la arteria basilar y ventilación mecánica
}

\author{
J. C. RODRÍGUEZ BORREGÁN, E. MIÑAMBRES, A. QUESADA SUESCUN \\ Departamento de Medicina Intensiva. Hospital Universitario Marqués de Valdecilla. \\ Santander
}

BASILAR ARTERY THROMBOSIS AND MECHANICAL VENTILATION

\section{RESUMEN}

Introducción: Conocer la incidencia, factores de riesgo, complicaciones y pronóstico de los pacientes con trombosis de la arteria basilar grave.

Material y métodos: Se revisaron de forma retrospectiva todos los pacientes diagnosticados de trombosis basilar que fueron conectados a ventilación mecánica en nuestro hospital durante un periodo de 10 años (11-1989 hasta el 31-12-1998). Se analizaron los siguientes datos: sexo, edad, factores de riesgo, causa de intubación, días de estancia en la UCI y en el hospital, el pronóstico a los 3 y 6 meses y las causas de fallecimiento.

Resultados: De los 24 casos encontrados, el 62,5\% fueron varones. La edad media entre los supervivientes y los fallecidos fue similar $(60,1$ \pm 16 y $62 \pm 15,8$ años). Los factores de riesgo más frecuentes fueron la hipertensión arterial, la fibrilación auricular, la diabetes mellitus y el tabaquismo. La estancia media en UCI fue 11,2 $\pm 14,8$ días y en el hospital 31,9 \pm 58,6 días. Las causas de intubación fueron: coma, protección de la vía aérea, parada cardio-respiratoria, crisis convulsivas generalizadas e infección respiratoria. La mortalidad fue del $75 \%$, siendo causada por progresión del coma o infecciones. El 16\% de los pacientes lograron una buena recuperación.

Conclusiones: Los pacientes que ingresaron en coma, en parada cardio-respiratoria reanimada y aquellos que presentaron crisis convulsivas generalizadas o neumonía tuvieron una mayor mortalidad. Se debe prestar especial atención a la aparición de hidrocefalia e infecciones por la importancia de un tratamiento precoz. En los pacientes con deterioro progresivo del nivel de conciencia la protección de la vía aérea quizás mejore el pronóstico.

PALABRAS CLAVE: Trombosis. Arteria basilar. Ventilación mecánica. Intubación orotraqueal. Mortalidad.

\section{ABSTRACT}

Background: To know the incidence, risk factors, complication and prognosis of the serious thrombosis of the basilar artery.

Methods: All patients diagnosed as having basilar thrombosis during a 10 year period (1/1/1989 to 31/12/1998) and having been connected to mechanical ventilation in our hospital are retrospectively revised. The following data are analised: gender, age, risk factors, cause of intubation, days of stay at the intensive care unit and at the hospital, the prognosis at 3 and 6 months and the causes of decease.

Results: $62.5 \%$ of the 24 found cases were males. The average age among the survivors and the deceased was similar $(60.1 \pm 16$ and $62 \pm$ 15.8 years). The most common risk factors were high blood-pressure, atrial fibrillation, diabetes mellitus and smoking. The average stay at the intensive care unit was $11.2 \pm 14.8$ days and $31.9 \pm 58.6$ days at the hospital. The cause of intubation were: coma, airway protection, cardiorespiratory arrest, general convulsive crisis and respiratory infection. Mortality reached $75 \%$ and was caused by progression of coma or infections. Only $16 \%$ of them recovered satisfactorily.

Conclusions: The patients admitted to hospital in coma, in revived cardiorespiratory arrest and those presenting general convulsive crisis or pneumonia have a higher mortality. Special attention must be drawn towards the appearance of hydrocephalus and infections due to the importance of an early treatment. In patient with a progresive damage of the consciousness level, the airway protection might improve the prognosis.

KEY WORDS: Thrombosis. Basilar artery. Mechanical ventilation. Orotracheal intubation. Mortality.

Rodríguez Borregán JC, Miñambres E, Quesada Suescun A. Trombosis de la arteria basilar y ventilación mecánica. An Med Interna (Madrid) 2001; 18: 525-528.

\section{INTRODUCCIÓN}

La trombosis de la arteria basilar representa el 20-26\% de todos los accidentes cerebrovasculares agudos de origen isquémico $(1,2)$, siendo una causa poco frecuente de muerte $(2$ casos cada 1.000 autopsias) (3) y que cursa con buen pronósti- co $(4,5)$. Sin embargo, cuando presenta una importante afectación neurológica se transforma en una enfermedad devastadora con pocas posibilidades de recuperación funcional y con una mortalidad superior al $70 \%$ (6-8).

En los últimos años se han realizado estudios con tratamiento fibrinolítico aunque todavía no hay resultados concluyentes.

Trabajo aceptado: 9 de Mayo de 2001

Correspondencia: Juan Carlos Rodríguez Borregán. Departamento de Medicina Intensiva. Hospital Universitario Marqués de Valdecilla. Avda. Marqués de Valdecilla s/n. 39008. Santander (Cantabria). 
Los objetivos de este trabajo han sido conocer en nuestra comunidad la incidencia, factores de riesgo y pronóstico de esta patología en los pacientes que requieren ventilación mecánica.

\section{MATERIAL Y MÉTODOS}

Se revisó de forma retrospectiva en nuestro hospital, que dispone del único Servicio de Cuidados Intensivos en esta Comunidad Autónoma de 525.000 habitantes, los pacientes que presentando una obstrucción aguda de la arteria basilar necesitaron ventilación mecánica. El periodo de estudio fue de 10 años (1 de enero de 1989 hasta el 31 de diciembre de 1998). El diagnóstico fue realizado por neurorradiólogos en 21 casos mediante la realización de tomografía axial computerizada (TAC), en 2 pacientes tras resonancia magnética nuclear (RMN) (casos 6 y 21) y en uno mediante Arteriografía (caso 4). Los pacientes recibieron tratamiento convencional con heparina sódica endovenosa para mantener un tiempo de tromboplastina parcial activada (TTPa) entre 1,5 y 2,5 veces el control, además también se les administró antiagregantes, excepto a los pacientes que presentaban alguna contraindicación. A dos pacientes (22 y 23) se colocó un drenaje ventricular por hidrocefalia. Se analizaron los siguientes datos: edad, sexo, causa de intubación, días de ventilación mecánica, estancia en UCI y en el hospital, los factores de riesgo de accidente cerebrovascular, puntuación en el Glasgow outcome scale (GOS) (9) a los 3 y 6 meses, la mortalidad y sus causas (Tabla I).

\section{RESULTADOS}

Durante estos diez años se han recogido 24 casos $(62,5 \%$ de sexo masculino), lo que representa una media de 2,4 casos/año, con un rango de 0 a 5 y una incidencia anual de 4,5 casos/millón de habitantes. La edad media fue de $62,1 \pm 15,9$ años con un rango de 26 a 85 ; siendo ligeramente mayor en el grupo de varones $(66,8 \pm 12,8)$ que en el de mujeres $(54,4 \pm$ 17,5). Los días de estancia media en UCI fueron $11,2 \pm 14,8$ y

TABLA I

\begin{tabular}{|c|c|c|c|c|c|c|c|}
\hline \multicolumn{8}{|c|}{ CARACTERÍSTICAS DE LOS PACIENTES ESTUDIADOS } \\
\hline № & $\begin{array}{l}\text { Edad/ } \\
\text { Sexo }\end{array}$ & $\begin{array}{l}\text { Factores de } \\
\text { riesgo }\end{array}$ & Causa de IOT & $\begin{array}{l}\text { Días de } \\
\text { lOT }\end{array}$ & $\begin{array}{l}\text { Estancia en } \\
\text { UCI/hospital }\end{array}$ & $\begin{array}{l}\text { GOS a los } \\
3 / 6 \text { meses }\end{array}$ & Causa de éxitus \\
\hline 1 & $26 / M$ & $\mathrm{ACO}$ & Coma & 11 & $20 / 24$ & $5 / 5$ & Neumonía \\
\hline 2 & $57 / \mathrm{H}$ & HTA, Tabaco, MD & PCR & 15 & $16 / 43$ & $5 / 5$ & Abdomen agudo \\
\hline 3 & $70 / \mathrm{H}$ & & Coma & 2 & $2 / 3$ & $5 / 5$ & Progresión del coma \\
\hline 4 & $34 / M$ & & $\begin{array}{l}\text { Deterioro del nivel } \\
\text { de conciencia }\end{array}$ & 2 & $5 / 24$ & $3 / 3$ & \\
\hline 5 & $74 / H$ & $\begin{array}{l}\text { HTA, DM , FA, } \\
\text { Hiperuricemia }\end{array}$ & PCR & 5 & $8 / 41$ & $2 / 2$ & \\
\hline 6 & $44 / H$ & & $\begin{array}{l}\text { Deterioro del nivel } \\
\text { de conciencia }\end{array}$ & 29 & $34 / 285$ & $3 / 3$ & \\
\hline 7 & $51 / \mathrm{H}$ & Tabaco & Coma & 2 & $2 / 2$ & $5 / 5$ & Progresión del coma \\
\hline 8 & $73 / \mathrm{H}$ & HTA, FA & Coma & 6 & $7 / 7$ & $5 / 5$ & Progresión del coma \\
\hline 9 & $57 / \mathrm{H}$ & HTA & PCR & 3 & $4 / 12$ & $5 / 5$ & Progresión del coma \\
\hline 10 & $36 / M$ & & Coma & 5 & $5 / 5$ & $5 / 5$ & Progresión del coma \\
\hline 11 & $83 / M$ & HTA & Coma & 12 & $13 / 14$ & $5 / 5$ & Progresión del coma \\
\hline 12 & $70 / \mathrm{H}$ & HTA & Infección respiratoria & 7 & $8 / 14$ & $5 / 5$ & Neumonía \\
\hline 13 & $42 / \mathrm{H}$ & Sd. Arnold-Chiari & Coma & 2 & $2 / 2$ & $5 / 5$ & Progresión del coma \\
\hline 14 & $56 / M$ & & Coma & 3 & $3 / 3$ & $5 / 5$ & Progresión del coma \\
\hline 15 & $71 / \mathrm{H}$ & HTA & PCR & 13 & $13 / 28$ & $5 / 5$ & Progresión del coma \\
\hline 16 & $59 / M$ & DM & $\begin{array}{l}\text { Deterioro del nivel } \\
\text { de conciencia + neumonía }\end{array}$ & 17 & $17 / 17$ & $5 / 5$ & Neumonía \\
\hline 17 & $66 / M$ & & PCR & 32 & $73 / 128$ & $4 / 5$ & Neumonía \\
\hline 18 & $72 / \mathrm{H}$ & HTA, IAO & Crisis generalizada & 6 & $6 / 15$ & $5 / 5$ & Progresión del coma \\
\hline 19 & $79 / \mathrm{H}$ & Tabaco & Coma & 3 & $3 / 3$ & $5 / 5$ & Progresión del coma \\
\hline 20 & $78 / \mathrm{H}$ & & $\begin{array}{l}\text { Crisis generalizada } \\
\text { +broncoaspiración }\end{array}$ & 2 & $2 / 42$ & $5 / 5$ & Sepsis abdominal \\
\hline 21 & $79 / H$ & HTA, DM , FA, MD, & $\begin{array}{l}\text { Deterioro del nivel } \\
\text { Hipercolesterolemia } \\
\text { de conciencia }\end{array}$ & 1 & $3 / 11$ & $2 / 2$ & \\
\hline 22 & $65 / M$ & HTA, FA & Coma & 2 & $4 / 7$ & $1 / 1$ & \\
\hline 23 & $65 / M$ & $\mathrm{FA}$ & $\begin{array}{l}\text { Deterioro del nivel } \\
\text { de conciencia }\end{array}$ & 7 & $11 / 26$ & $2 / 2$ & \\
\hline 24 & $85 / H$ & $\mathrm{FA}$ & Coma & 3 & $9 / 10$ & $5 / 5$ & Neumonía \\
\hline
\end{tabular}

M: mujeres, $\mathrm{H}$ : varones, ACO : ingesta de anticonceptivos orales, PCR: parada cardiorrespiratoria, M D: miocardiopatía dilatada, HTA: hipertensión arterial, DM : diabetes mellitus, FA: fibrilación auricular, IAO: insuficiencia aórtica. IOT: intubación orotraqueal. 
en el hospital de $31,9 \pm 58,6$, con un rango de 2 a 73 y de 2 a 285 días respectivamente. La mortalidad fue del $75 \%$, con una edad media en los fallecidos $(62,8 \pm 15,8$ años $)$ similar a la del grupo de supervivientes $(60,1 \pm 16$ años). Se consideró una buena recuperación a los pacientes que alcanzaron la independencia funcional (Glasgow Outcome Scale $\leq 2$ ), consiguiéndose en un $16 \%$ de los casos.

Los pacientes que ingresaron en coma $(n=11)$ presentaron una mortalidad del $91 \%$, con un superviviente ( $\left.n^{\circ} 22\right)$, que presentó una buena recuperación funcional. En el subgrupo que ingresó en parada cardio-respiratoria $(n=5)$, la mortalidad fue del $80 \%$, con un único superviviente $\left(n^{\circ} 5\right)$ el cual presentó una aceptable recuperación funcional. Los dos pacientes que fueron intubados por presentar una crisis convulsiva generalizada (casos 18 y 20) y los tres que en el momento de ser intubados presentaban una neumonía por aspiración (casos 12, 16 y 20) fallecieron. En los 5 pacientes que se intubó para proteger la vía aérea por deterioro progresivo del nivel de conciencia (con una puntuación en la escala de coma de Glasgow entre 10 y 12 puntos) sólo hubo un fallecimiento $\left(\mathrm{n}^{\mathrm{o}} 16\right.$, que además ya presentaba neumonía en el momento de ser intubado), lo que supone una mortalidad del $20 \%$.

En nuestra serie la causa de fallecimiento más frecuente con el $61 \%(11 / 18)$ fue la progresión del coma hasta llegar a la muerte cerebral, seguida por la neumonía con el $27 \%(5 / 18)$ y en último lugar por infecciones abdominales con el $11 \%$ (2/18).

Los factores de riesgo encontrados fueron: la hipertensión arterial en 10 pacientes (41\%), la fibrilación auricular en 6 (25\%), la diabetes mellitus y el tabaco en $3(12,5 \%)$, miocardiopatía dilatada en $2(8,3 \%)$ y en un caso cada uno $(4,2 \%)$ hipercolesterolemia, hiperuricemia, insuficiencia aórtica, síndrome de Arnold-Chiari e ingesta de anticonceptivos orales. En 7 pacientes (29\%) no se registraron factores de riesgo.

\section{DISCUSIÓN}

Nuestra serie presenta una mortalidad sensiblemente inferior que las de Wijdicks (6), Hacke (8) y similar a la de Archer (7) (Tabla II). El porcentaje de pacientes que alcanzaron la independencia funcional $(\mathrm{GOS} \leq 2)$ fue similar a la de Hacke (8), siendo ambas superiores a las otras dos en las que nadie lo logró. Los pacientes que presentando una oclusión aguda de la arteria basilar necesitan ser conectados a ventilación mecánica presentan una elevada mortalidad. En nuestra serie el coma, la parada cardio-respiratoria reanimada, las crisis convulsivas generalizadas y la broncoaspiración empeoraron el pronóstico.

\section{TABLA \|}

SERIES DE TRO M BO SIS BASILAR CO N IM PO RTANTE AFECTACIÓ N NEURO LÓ GICA

\begin{tabular}{|c|c|c|c|}
\hline & $\begin{array}{c}\text { W ijdicks (6) } \\
(n=25)\end{array}$ & $\begin{array}{l}\text { Archer (7) } \\
(n=20)\end{array}$ & $\begin{array}{c}\text { Hacke (8) } \\
(n=22)\end{array}$ \\
\hline Tratamiento & Heparina e.v. & ND & $\begin{array}{l}\text { Heparina y/o } \\
\text { antiagregantes }\end{array}$ \\
\hline M ortalidad & $88 \%$ & $75 \%$ & $86 \%$ \\
\hline Buena recuperación & $n * \quad 0 \%$ & $0 \%$ & $14 \%$ \\
\hline
\end{tabular}

ND: no hay datos; e.v.: endovenoso; *GOS $\leq 2$
En los ensayos con tratamiento fibrinolítico la mortalidad de los pacientes que están en coma es muy elevada (77-83\%) $(10,11)$, por lo que se discute su indicación. Sin embargo, con el tratamiento convencional la mortalidad es superior al $90 \%$ por lo que creemos que este subgrupo aunque empeore los resultados globales no debe ser excluido de estos protocolos.

Al observar que el $66 \%$ de estos enfermos acuden a urgencias en coma o en parada cardio-respiratoria junto con la dificultad en su diagnóstico nos hace pensar que quizás más enfermos de los que acuden por estos motivos presenten esta patología sin llegar a ser diagnosticada, estando infravalorada su incidencia y mortalidad. Creemos que se debe prestar atención a la aparición de complicaciones que frecuentemente se asocian a esta patología, por la importancia del tratamiento precoz, como son la hidrocefalia (casos 22 y 23 que alcanzaron una buena recuperación funcional tras realizarles drenaje ventricular), las infecciones y la aparición de crisis convulsivas, aunque esta última más que complicación sea una expresión de gran daño neurológico. Los pacientes fallecidos, excepto el caso $\mathrm{n}^{\circ} 17$, lo fueron en los 3 primeros meses (el $75 \%$ en los 15 primeros días), siendo además este el único paciente que ha variado su puntuación en el GOS de los 3 y 6 meses, por tanto de los pacientes que alcanzaron los 6 meses de supervivencia ninguno mejoró el grado de recuperación funcional alcanzado a los 3 meses.

La HTA fue el factor de riesgo más frecuente, estando presente en casi la mitad de nuestros enfermos. La presencia de fibrilación auricular, que sugiere un perfil embólico del ictus (aunque en 4 de estos pacientes también hay otros antecedentes que podrían apoyar el perfil trombótico), fue observada en todos los pacientes que sobrevivieron con buena recuperación $(\mathrm{GOS} \leq 2)$. En el subgrupo de enfermos con fibrilación auricular sólo hubo dos fallecidos (33\%), muy por debajo de la mortalidad global, a pesar de tener una edad media superior al resto de pacientes (73,5 \pm 7,1 contra 58,3 $\pm 16,2$ años). Sin embargo, el tamaño de la muestra no permite sacar conclusiones.

De los pacientes que fueron intubados para proteger la vía aérea $(n=5)$, sólo falleció uno $\left(n^{\circ} 16\right)$ que ya tenía una neumonía, el resto no presentó ninguna complicación asociada a la ventilación mecánica, por lo que pensamos que no se debe retrasar esta medida. El pronóstico de quienes sobreviven a un ictus vertebrobasilar se ve afectado fundamentalmente por las complicaciones neurológicas (recurrencia del ictus), cardiacas (IAM e ICC) e infecciosas (3). En nuestra serie todos los enfermos fallecidos lo fueron por progresión del coma o por infecciones.

Es necesario pensar en esta patología en aquellos pacientes que acuden a urgencias en coma sin filiar y en parada cardio-respiratoria, aunque no presenten factores de riesgo.

Creemos que es importante la intubación precoz para proteger la vía aérea en los pacientes que presentan deterioro progresivo del nivel de conciencia.

Las causas de fallecimiento fueron la progresión del coma y las infecciones respiratorias o abdominales. Pensamos que se debe prestar una atención especial a la aparición de hidrocefalia e infecciones por la importancia de un tratamiento precoz.

En nuestra serie los pacientes con fibrilación auricular tienen mejor pronóstico, a pesar de tener una edad media sensiblemente superior.

Los enfermos que ingresan en coma o en parada cardiorespiratoria junto con los que presentan broncoaspiración o crisis convulsivas generalizadas presentan un peor pronóstico, con una mortalidad superior al $80 \%$. 


\section{Bibliografía}

1. Turney TM, Garraway WM, Whisnant JP. The natural history of hemispheric and brainstem infaction in Rochester, Minnesota. Stroke 1984; 15: 790-794.

2. Bogousslavsky J, Van Melle G, Regli F. The Lausane Stroke Registry: analysis of 1.000 consecutive patients with first stroke. Stroke 1988; 19 : 1083-1092.

3. Becker KJ, Purcell LL, Hacke W, DF Hanley. Vertebrobasilar thrombosis: Diagnosis, management, and the use of intra-arterial thrombolytics. Crit Care Med 1996; 24: 1729-1742.

4. Caplan LR. Posterior Circulation Disease: Clinical Findings, Diagnosis, and Management. Cambrigde, Mass: Blackwell Scientific Publications; 1996.

5. Bogousslavsky J, Regli F, Maeder P, Meuli R, Nader J. The etiology of posterior circulation infarcts: a prospective study using magnetic resonance imaging and magnetic responance angiography. Neurology 1993; 43: $1528-1533$.
6. Wijdicks EF, Scott P. Outcome in patients with acute basilar artery occlusion requiring mechanical ventilation. Stroke 1996; 27: 13011303.

7. Archer CT, Horenstein S. Basilar artery occlusion. Clinical and radiological correlation. Stroke 1977; 8: 383-387.

8. Hacke W, Zeumer H, Ferbert A, Bruckmann H, del Zoppo GJ. Intraarterial thrombolytic therapy improves outcome in patients with acute vertebrobasilar occlusive disease. Stroke 1988; 19: 1216-1222.

9. Jennett B, Bond M. Assessment of outcome after severe brain damage: a practical scale. Lancet 1975; 1: 480-484.

10. Brandt T, Von Kummer R, Muller-Kuppers M, Hacke W. Thrombolytic therapy of acute basilar artery occlusion. Variables affecting recanalización and outcome. Stroke 1996; 27: 875-881.

11. Huemer M, Niederwiesser V, Ladurner G. Thrombolytic treatment for acute occlusion of the basilar artery. J Neurol Neurosurg Psychiatry 1995; 58: 227-228. 